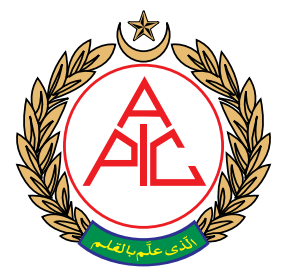

Anesthesiology Department, Centro Hospitalar e Universitário de Coimbra, (Portugal)

Ana Sampaio [anasssampaio@gmail. com]

Gustavo Norte [gustavo.norte@gmail. com]

Liliane Godinho [liliane godinho@ hotmail.com]

Ana Raimundo [ana.s.raimundo@ gmail.com]

Manuel Cuervo [manuelteijeiro@ yahoo.es]

Correspondence: Ana Sofia Silva Sampaio,

Urbanização Panorama, Lote 9 ,

Cave esquerda 3000-446 Coimbra, (Portugal)

E-mail: anasssampaio@gmail.com Phone: +351 937609429

Received: 24 September 2019,

Reviewed: 24, 26 October 2019,

Accepted: 22 November 2019

\section{A case report of a complicated fatal percutaneous valve implantation}

\author{
Ana Sampaio, Gustavo Norte, Liliane Godinho, Ana Raimundo, \\ Manuel Cuervo
}

\begin{abstract}
Pulmonary valve dysfunction, also called right ventricle outflow tract dysfunction, is one of the known anomalies in patients with congenital heart disease. Percutaneous pulmonary valve implantation (PPVI) has been developed as an alternative to surgical correction. Although it is largely considered a safe procedure, life-threatening complications can happen and institutions must be able to resolve these immediately and adequately.
\end{abstract}

We present a case of pulmonary valve migration to the right ventricle during a PPVI. This complication needed immediate cardiac surgery due to resulting hemodynamic instability. The percutaneous valve prosthesis was removed and a bioprosthetic valve was surgically implanted in the correct position.

This case emphasizes the need of prompt cardiac surgery support and the readiness of the anesthesia team to deal with emergency open cardiac surgery.

Key words: Pulmonary Valve; Heart Valve Prosthesis Implantation; Cardiac Surgical Procedures

Citation: Sampaio A, Norte G, Godinho L, Raimundo A, Cuervo M. A case report of a complicated fatal percutaneous valve implantation. Anaesth pain \& intensive care 2019;23(4):398-400, DOI: 10.35975/apic.v23i4.1174

\section{INTRODUCTION}

The incidence of congenital heart disease ranges from 5-8 per thousand live births. In adults, the estimated prevalence is $1 / 150$ people. Approximately $20 \%$ of newborns with congenital heart disease have anomalies in the pulmonary valve or right ventricular outflow tract (RVOT), such as tetralogy of Fallot, truncus arteriosus or pulmonary atresia. ${ }^{1}$

Usually, surgical treatment of these patients requires repeated interventions due to progressive dysfunction of the RVOT. Although the mortality rate associated with surgical treatment is low, morbidity is significant. Therefore, in order to achieve a balance between the risks associated with RVOT dysfunction and the need to minimize the total number of surgeries throughout life, it has become imperative to find less invasive nonsurgical alternatives. Thus, percutaneous pulmonary valve implantation (PPVI) was developed for the treatment of RVOT dysfunction, being considered a safe and effective alternative. ${ }^{1,2}$

PPVI was the first percutaneous intervention for treatment of regurgitant lesions in humans. ${ }^{3}$ This technique allows RVOT gradient normalization and resolves pulmonary regurgitation immediately after implantation. It is also associated with significant improvements in right ventricle (RV) functional capacity and systolic function. In addition, the leftsided systolic function also improves, with increased left ventricle (LV) systolic volume, aortic pressure and left ventricular ejection fraction (LVEF) ${ }^{1,2}$ These hemodynamic benefits can be observed in medium and long term.

Due to the lack of long-term data, it is not currently known if all patients submitted to PPVI will require repeated procedures, as shown in surgical cases. Knowing that this procedure has risks and complication, it is particularly important to assure access to proper surgical and hemodynamic suites to mitigate these. We present this case report to 
emphasize the need of availability of prompt cardiac surgery support and the cardiac anesthesia team to deal with emergency open cardiac surgery if such need arises

\section{CASE REPORT}

A 45-year-old female patient was admitted to cardiac surgery operating room (OR) after a complicated PPVI. She was submitted to tetralogy of Fallot correction in 1987 and had a homograft pulmonary valve placement in 1992 .

By presenting severe pulmonary regurgitation, homograft calcification and an infundibular region with $21 \mathrm{~mm}$, she was proposed to undergo PPVI.

In the hemodynamic suite (catheterization laboratory), a covered CP stent with $45 \mathrm{~mm}$ and a Melody valve $22 \mathrm{~mm}$ were implanted percutaneously in pulmonary position. During the procedure, she developed hemodynamic instability with monomorphic ventricular tachycardia due to migration of the prosthesis to the right ventricle. After four synchronised shocks and a loading dose of amiodarone, sinus rhythm was restored. Due to severe hypotension, a norepinephrine infusion was initiated.

She arrived at the cardiac OR conscious and oriented, with no signs of respiratory distress and hemodynamically stable with norepinephrine infusion.

General anesthesia was initiated with fentanyl, etomidate and rocuronium and maintained with sevoflurane (except during cardiopulmonary bypass when propofol was used), in association with remifentanil and rocuronium infusions.

The percutaneous valve prosthesis (Melody valve) was removed and a bioprosthesis was surgically implanted in pulmonary position. During the surgery, hemodynamic stability was assured with norepinephrine and no complications were observed. After surgery she was transported to the cardiac care unit (CCU).

On the day after surgery, she developed left lower limb compartment syndrome which necessitated fasciectomy and repair of her left femoral artery, where the vascular access for PPVI had been performed. She was extubated $30 \mathrm{~h}$ after the pulmonary valve implantation with no other complications.

On the third postoperative day, she developed dyspnoea and desaturation. She was reintubated and mechanically ventilated. During transport preparation to perform an angio-computerized tomography due to pulmonary thromboembolism assumption, she developed cardiac arrest. Despite prompt cardiopulmonary resuscitation manoeuvres, she could not be saved.

\section{DISCUSSION}

PPVI has become an attractive alternative to surgery in patients with dysfunctional RVOT, being one of the most interesting recent developments in the treatment of structural heart disease. ${ }^{2}$ A 2011 scientific statement from the American Heart Association (AHA), American Academy of Pediatrics (AAP) and Society for Angiography and Cardiovascular Interventions (SACI) has granted a Class IIa (Level of Evidence B) recommendation for PPVI in patients with RVOT dysfunction in the presence of moderate or severe pulmonary regurgitation or stenosis. ${ }^{2}$ The European Society of Cardiology (ESC) and Association for European Pediatric Cardiology (AEPC) recommend PPVI with the same indications as surgical pulmonary valve replacement. ${ }^{2}$

Active infection and pregnancy are absolute contraindications for this procedure. ${ }^{2}$ Patients weighing less than $20 \mathrm{~kg}$, with vascular injury or cardiac trauma are at increased risk of complications. ${ }^{3}$

PPVI was not directly compared with the surgical intervention, however it has been accepted as an alternative therapeutic procedure for its safety and effectiveness with low mortality, which is more related to patient comorbidities than to the procedure itself. It can avoid or delay the surgical intervention and associated morbidities. The absence of reintervention after PPVI was reported $>90 \%$, $>80 \%$ and $>70 \%$ at 1,2 and 4 years, respectively. ${ }^{1}$ The most common cause for reintervention with the Melody valve is stent fracture (5-16\%). ${ }^{2}$ The risk of infective endocarditis has been reported between $1 \%$ and $4 \%$ with the Melody valve. ${ }^{2}$

In the US Melody study, the rate of serious complications was $6 \%$, including death from coronary dissection, rupture of the conduit, unstable arrhythmia, perforation in the distal pulmonary artery, and femoral vein thrombosis. Migration of the valve or stent occurred in four patients, three of whom required surgical removal. ${ }^{2}$ In cases of embolization or valve migration, surgical removal is the most controlled approach due to the risk of right ventricle and tricuspid valve injury. ${ }^{3}$

Other complications described include death, stent fracture, coronary impingement, peri-valvular leakage, thromboembolism and stenosis or valve regurgitation. ${ }^{2}$

Although percutaneous implantation of cardiac valves is considered a safe and effective non-surgical therapeutic with high success rate, as an alternative to surgical valve replacement, embolization or valve 
migration is one of the possible complications of this procedure and may require emergency surgical treatment as it happened in our reported case.

In addition, as Covello, et al. ${ }^{4}$ state with regard to transcatheter aortic valve implantation (TAVI), all patients undergoing this procedure should remain in an CCU for at least 24 hours and under close surveillance for several days, especially regarding hemodynamic stability, vascular access, dysrhythmias and renal function. Although recovery is often quick, simple and without complications, where early transfer to a telemetry-equipped intermediate care unit may be an appropriate strategy in selected patients, we should take into account that they are high-risk patients predisposed to complications at any time during hospitalization, with a complication pattern substantially different from standard cardiac surgery. ${ }^{4}$ Taking this into account and considering that this kind of complications lead to life-threatening conditions associated with hemodynamic instability, the need of transport to a cardiac surgery centre may carry unnecessary increased risk.

We conclude that this type of procedures should be performed in centres that can provide prompt cardiac surgical support.

Conflict of interest: None declared by the authors

Authors' contribution:

AS, GN - case report writing

LG, AR - manuscript editing

AR - manuscript editing

MC - anaesthetist in the clinical case, manuscript editing

\section{REFERENCES}

1. Ansari MM, Cardoso R, Garcia D, Sandhu S, Horlick E, Brinster D, et al. Percutaneous pulmonary valve implantation: present status and evolving future.J Am Coll Cardiol. 2015 Nov 17;66(20):2246-55. [PubMed] DOI: $10.1016 /$ i.jacc. 2015.09 .055

2. Suradi HS, Hijazi ZM. Percutaneous pulmonary valve implantation. Glob Cardiol Sci Pract. 2015;2015(2):23. [PubMed] DOI: $\underline{10.5339 /}$ gcsp.2015.23

3. Khambadkone S. Percutaneous pulmonary valve implantation. Ann Pediatr Cardiol. 2012;5(1):53-60. [PubMed] DOI: 10.4103/0974- $\underline{2069.93713}$

4. Covello RD, Landoni G, Michev I, Bignami E, Ruggeri L, Maisano F, et al. Percutaneous aortic valve implantation: the anesthesiologist perspective. HSR Proc Intensive Care Cardiovasc Anesth. 2009;1(2):28-38. [PubMed] 